\title{
La Etología del niño y el estudio de los sistemas de comunicación no verbales
}

\section{Rodríguez, H. Montagner, A. Restoin, B. Schaal, $\mathrm{V}$ : Ullmann*:}

Universidad de Besanfon

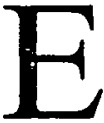

L estudio del comportamiento humano ha sido emprendido desde hace mucho tiempo por los psicólogos, algunos psiquiatras y psicoanalistas (J. Piaget, H. Wallon, S. Freud, R. Spizt, J. Bowlby, etc.), habiendo permitido dicho estudio conocer ciertos aspectos importantes del desarrollo del niño.

Pueden compararse estos estudios con aquellos de los etólogos, quienes desde los años 1967-1968 construyen el repertorio de las unidades de comportamiento del niño. Ciertos etólogos como l. EiblEibesfeldt de la Universidad de Munich, buscan en dicho repertorio las unidades de comportamiento que pertenecen al patrimonio genético de la especie humana. Otros hacen simplemente una descripción refinada de las unidades de comportamiento $y$ de su combinatoria sin pronunciarse sobre su determinismo (N. G. Blurton Jones, 1972; W. C. Mac Grew, 1972; J. C. Rouchouse, 1978). Sin embargo, se puede desprender de estos diferentes pasos y métodos etológicos un punto de convergencia importante: el estudio del comportamiento del niño como respuesta a los estímulos de su ambiente social y fisico en -el transcurso de actividades libres, en situaciones no apremiantes.

Los etólogos del niño completan así las investigaciones de la Psicologia experimental que a menudo sitúa sus observaciones dentro de situaciones estandarizadas y en laboratorio. El objetivo del etólogo es el llegar a discernir, dentro de las condiciones habituales de vida de la especie, la parte que corresponde a los factores exógenos (factores ecológicos y factores sociales) y a los factores endógenos (factores fisiológicos, experiencias individuales) en la expresión de un comportamiento.

La Etología del niño se destaca de esta manera como una disciplina

- Dirección de los autores: Laboratorio de Psicofisiologia. Departamento de Biologia del Comportamiento y Ecologia. Facultad de Ciencias y de Téenicas. Route de Gray. 29030 Besançon, Francia. 
de conexión entre los naturalistas (especialistas de la Etologia animal y de la Ecología), los psicólogos, los psiquiatras y los lingüistas que estudian el desarrollo del niño, pero también entre los naturalistas y los neurobiólogos que estudian la génesis de las estructuras y del funcionamiento del sistema nervioso. La Etologia contribuye a dar su verdadero sentido a la noción de adaptación cuando ésta se define como la capacidad de respuesta y de diálogo entre el organismo y su medio ambiente fisico y social.

De manera general la Etologia humana es la disciplina que completa las investigaciones de los lingüistas mediante el estudio de los modos de comunicación que no están ligados directamente al discurso (mimicas, posturas, gestos, contactos corporales, olores, vocalizaciones). A imagen de la Etología animal, las observaciones y análisis se realizan dentro de los marcos habituáles de vida del niño o del adulto, tomando en cuenta el contexto, las experiencias anteriores, el estado y la evolución fisiológica del emisor y del receptor de la información. No siendo una disciplina reduccionista ni determinista, la Etologia humana dota de medios metodológicos y conceptuales a todas las ciencias que estudian al hombre dentro de su medio ambiente ecológico y social, desde el nacimiento hasta la muerte.

Dentro de esta perspectiva, nuestro grupo de investigación dirigido por el profesor.H. Montagner, estudia la manifestación y la evolución de los sistemas de percepción, de los comportamientos relacionales y de los ritmos biológicos.

\section{MATERIAL Y METODOS DE ESTUDIO}

Habiendo emprendido desde 1970 los estudios sobre la génesis de los sistemas de comunicación, el grupo de investigación de la Universidad de Besançon ha adoptado, desde el principio, una actitud funcionalista. Mediante la observación en continuo, la toma de apuntes, la grabación de imágenes y la grabaciōn sonora (cámara fotográfica, filmadora, sistema video y grabadora), hemos, en primer lugar, desprendido los comportamientos que inducen respuestas caracteristicas en el niño receptor.

Se han buscado luego las correlaciones eventuales entre las modificaciones de dichos comportamientos y una serie de variables (edad, sexo, acontecimientos fisiológicos, familiares, composición del grupo). Paralelamente, unos análisis más refinados (análisis de péliculas imagen tras imagen $y$ análisis espectral de las vocalizaciones) nos han permitido discernir progresivamente cómo se manifiestan las más pequeñas unidades de comportamiento (llamadas «items») y cómo se combinan en el transcurso del desarrollo, para formar los comportamientos más complejos que regulan las interacciones del niño con sus semejantes.

Tales estudios implican la adaptación de protocolos experimentales que correspondan a los objetivos establecidos por la investigación y al nivel de desarrollo del niño ( $H$. Montagner y col. 1973-1983). Las investigaciones se realizan dentro de estructuras existentes y que se prestan al estudio de las diferentes categorias de edad. Cuatro poblaciones de niños se estudian separadamente: los recién nacidos en la clinica obstétrica, los niños de 6 meses a 3 años en la guarderia, los 
niños de 3 a 6 años en el jardin de infancia y los niños de 6 a 12 años en la escuela primaria. En total, y desde el año 1970 han sido observados más de 1.200 niños.

\section{Ontogenia de las discriminaciones y de los estimulos especificos entre la madre y el lactante}

Si la ontogenia de las discriminaciones olfativas, visuales y auditivas no especificas ha sido particularmente bien estudiada en el bebé humano (L. P. Lippsit, 1977; J. E. Steiner, 1979; M. Russel, 1976; T. G. R. Bower, 1974-1978; J. Melher, 1978; E. Vurpillot, 1972; C. Trevarthen, 1978), aquella de las discriminaciones especificas (olfativas, gustativas y somestésicas) ha sido a menudo menospreciada. Y, sin embargo, las investigaciones etológicas han demostrado la importancia de dichos estimulos, particularmente de los olores, en la formación de los vínculos privilegiados entre el joven mamifero y su madre -en este caso los olores desempeñan el papel de feromonas (móléculas químicas producidas por las glándulas y secretadas hacia el exterior).

Hasta 1974 no se publican los-primeros estudios sobre las diferencias de comportamiento observadas en los lactantes, en presencia del olor de sus madres o del olor de madres ajenas. A. MacFarlane (1975) ha demostrado efectivamente, mediante un método etológico, que desde el sexto dia post-natal, el lactante orienta la cabeza de manera preferencial hacia un apósito de gasa impregnado del olor del seno materno. H. Montagner demostraba paralelamente que los niños de 28 a 36 meses que tenían que elegir entre dos camisetas impregnadas del olor de madres distintas elegian de manera significativa la camiseta materna (H. Montagner, 1974). Se dispone actualmente de datos más precisos sobre la discriminación del olor materno por el lactante gracias a la adaptación por nuestro equipo de un método original de cuantificación de los movimientos de orientación de la nariz y de los dos brazos del recién nacido, según que éste se halle confrontado con olores corporales específicos (del seno o del cuello de la madre o de madres ajenas) o ante situaciones desprovistas de todo olor especifico (B. Schaal el al., 1980-1983).

\section{Protocolo experimental}

- Varias madres se colocan al mismo tiempo unos apósitos de gasa, durante uno o dos dias, sobre los senos (colecta de secreciones lácteas, secreción de las glándulas"sebáceas y de las glándulas sudoriparas del pezón y del seno) y sobre el cuello (colecta de secreciones sebáceas y sudoriparas).

- En cada experiencia dos de dichos apósitos se colocan en las dos ramas de un dispositivo en forma de "U»" entre las cuales se sitúa la cabeza del niño (Fig. I).

- El experimentador pone la nariz del niño en contacto con el apósito proveniente de una madre extraña (E), luego con aquel de su madre $(M) e$ inversamente.

- Después de haber situado la cabeza del niño entre los dos tapones, se filma su comportamiento durante 1 o 2 minutos. 


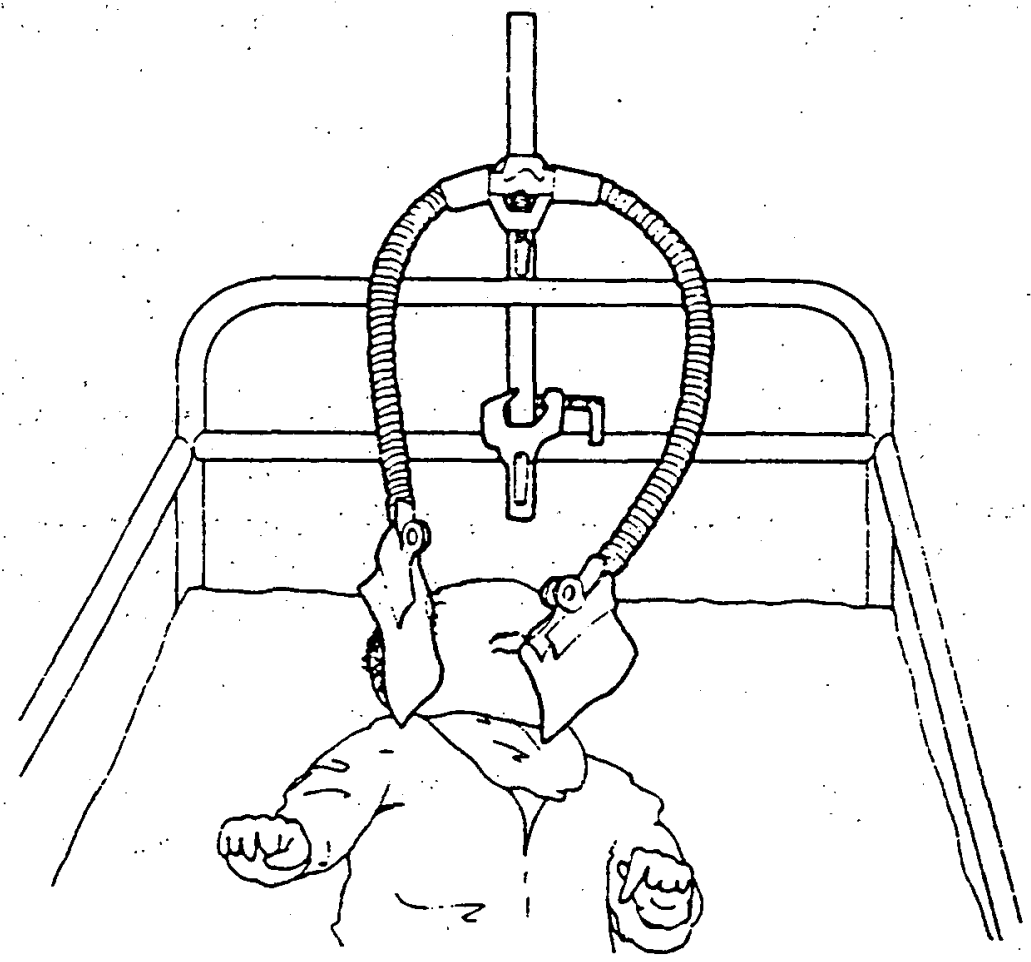

Figura 1

Dispositivo experimental que permite el estudio del comportamiento del recién nacido. Su nariz se encuentra en contacto con un apósito de gasa impregnado de olores maternos 0 un apósilo de gasa impregnado de olores de una madre extraña o un apósito olfativamente "neutral".

- Luego se permutan los dos apósitos y se filma nuevamente el comportamiento del lactante durante 1 . 02 minutos.

Una vez reveladas, las peliculas son analizadas imagen tras imagen: las posiciones de la nariz y de los dos brazos son señaladas cada dos segundos en una hoja transparente colocada directamente sobre la pantalla de un visualizador. Se calca luego dicha hoja sobre un papel milimerrado, lo que permite calcular la extensión y la densidad de los movimientos de la nariz y de los dos brazos. Se obtiene de esta mariera un «kinegrama» de cada experiencia y para cada niño (Fig.' 2 ).

\section{Resultados}

El análisis «kinegráfico», reconstruido gracias a la visualización de las peliculas, ha demostrado que desde el tercer dia el comportamiento del bebé se halla significativamente modificado cuando su nariz se encuentra en presencia de olores maternos $(M)$ con respecto a las situaciones en las que se halla en presencia de olores de una madre extraña (E) o de un olor no especifico (Fig. 3).

Paralelamente, desde el tercer día la madre reconoce el olor de su bebé de manera significativa ( $\ 60$ por 100 ) entre otros olores provenientes de otros lactantes ( $p r u e b a X^{2}, p .<0,01$ ). Dicho reconocimiento 


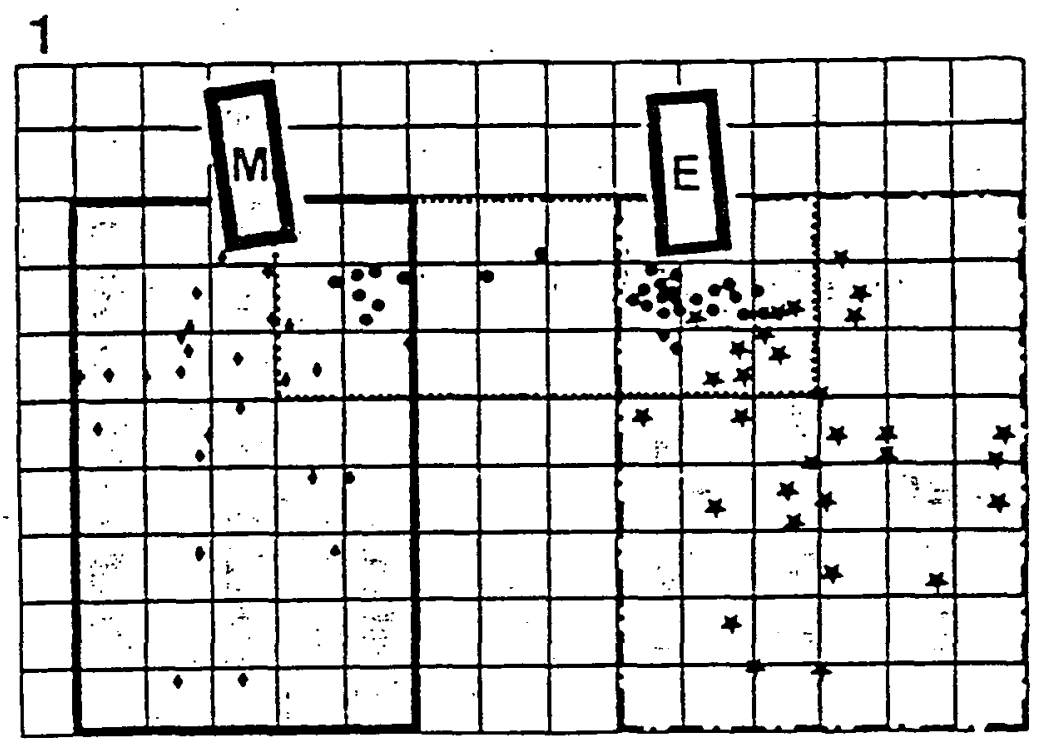

2

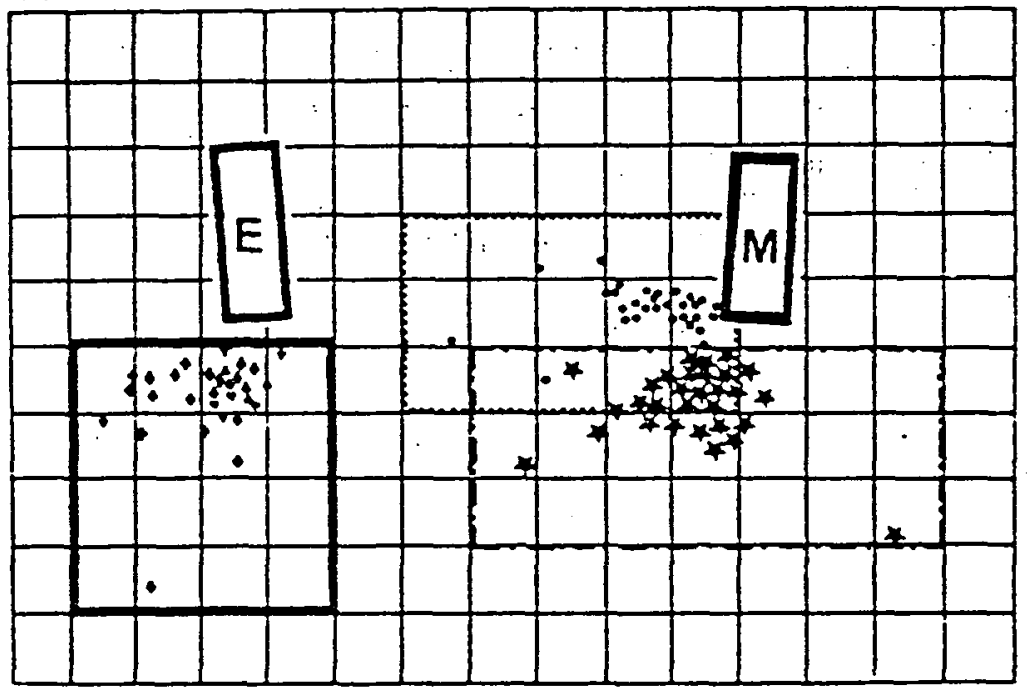

- .. Posiciones de la nariz

.... Extensión de los movimientos de la nariz

$\star \quad$ Posiciones del brazo izquierdo

.-. Extensión de los movimientos del brzzo izquierdo

$+\quad$ Posiciones del brazo derecho

- Extensión de los movimientos del brazo derecho

FIGURA 2

Ejemplo de un "kinegrama» mediante el cual se realiza un estudio cuantitativo de las posiciones y de los movimientos de la narizy de los brazos del lactante. La nariz se balla en contacto con un tapón de gasa impregnado de olores maternos (secreciones lácteas + secreciones de las gländulas sudoriparas y sebaceas) (M) y un tapón de gasa impregnado de olores de otra madre (E). Las posiciones de la narizy de cada brazo están indicadas 


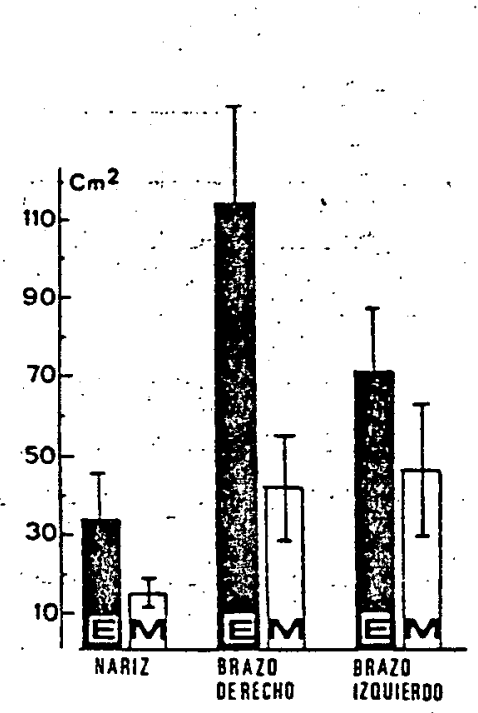

A

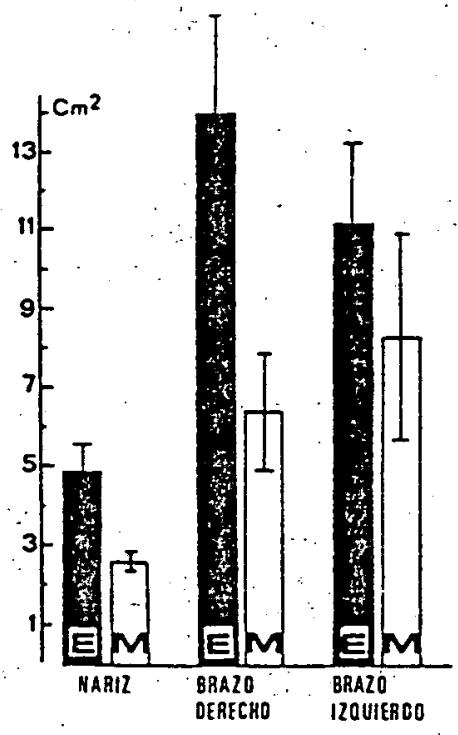

FIGURA ;

Extension $(A)$ y densidad $(B)$ de los movimientos de la nariz $y$ de los dos brazos de $s$ lactantes de 3 a 10 días, a los cuales se les ba presentado sucesivamente, a un minuto de intervalo, en tapón de gasa impregnado del alor del cuello materno (M) y un tapón de gasa impregnado del olor del cuello de una madre extraria $(E)$. Cada media está representada con un error estandar.

aparece aún en el segundo día cuando el contacto corporal (piel a piel) entre la madre y el recién nacido es de larga duración. Sin embargo, la curva de reconocimiento del olor del bebé por la madre presenta una evolución característica del tercero al décimo dia postnatal, con la particularidad de una baja entre el cuarto y el sexto dia. La curva de reconocimiento acústico (grabación de los lloros de varios bebés) del lactante por la madre sigue una evolución similar entre el tercero y el décimo día (Fig. 4).

La búsqueda de correlatos fisiológicos demuestra que si la tasa de estrógenos totales, de estriol y de pregnandiol en las orinas de la madre baja desde el segundo dia y se estabiliza al cuarto día, la tasa de los 17-hidroxicorticosteroides o 17 -OHCS (hormonas esteroides de defensa) urinarios maternos presenta una evolución temporal invertida con respecto a las curvas de reconocimiento olfativo y acústico (Fig. 4).

Estas investigaciones sobre el recién nacido demuestran que sólo el estudio ontogenético del conjunto de las capacidades de discriminación sensoriales del lactante y de todos los tipos de interacción posibles con su madre nos permitirá una mejor comprensión del papel respectivo de los diferentes tipos de estímulos exógenos a partir de los cuales. se construyen, se refuerzan o se deterioran los intercambios entre la madre y el bebé en tal o cual etapa de la vida postnatal. Se ha podido asi demostrar que los primeros vinculos entre la madre y el lactante se establecen, además de en otros aspectos, por intermedio del reconocimiento mutuo del olor corporal. Encontramos de esta manera un mecanismo de vínculo entre madre y cria muy propagado en los 


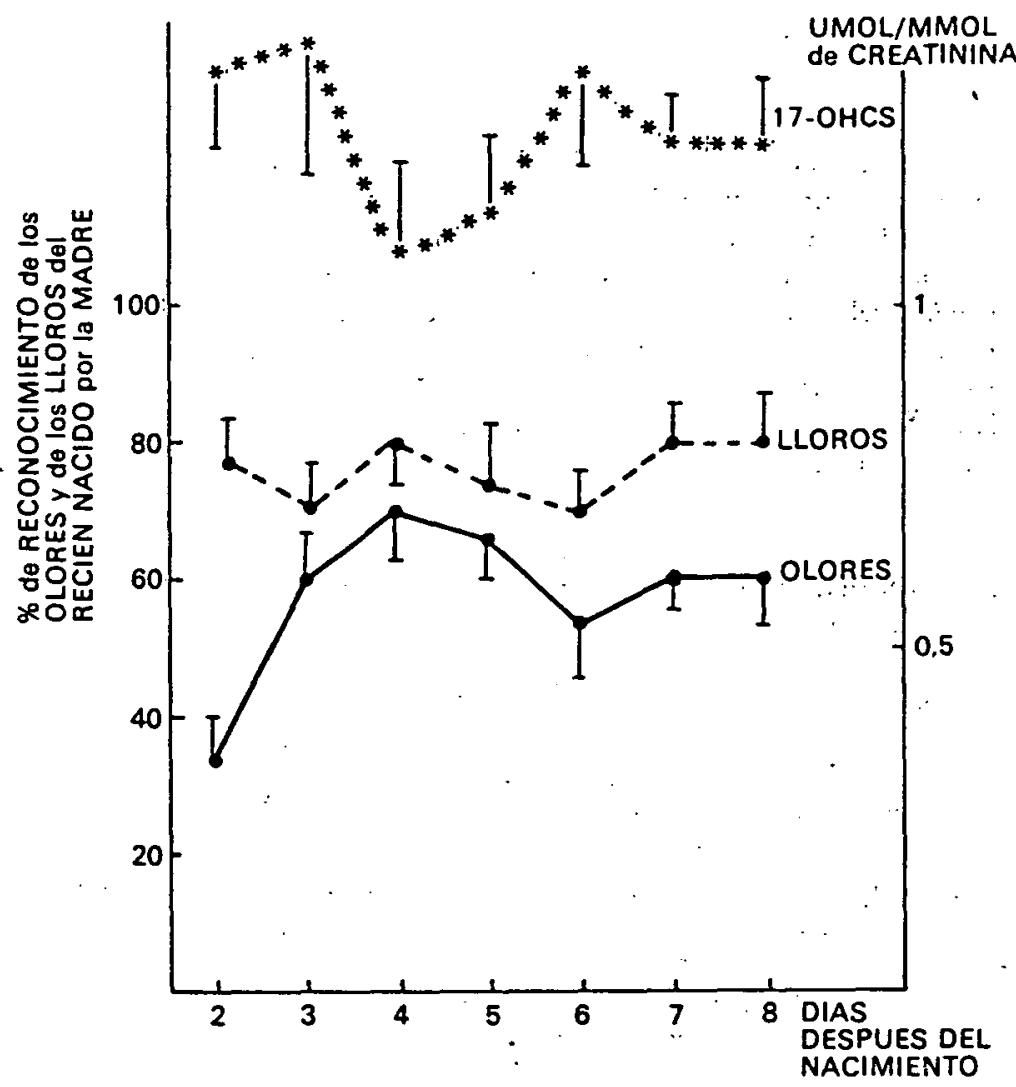

FigurA 4

Evolucion del porcentaje de reconocimiento por la madre del olor corporal (pruebas efectuadas con 20 madres) y de los lloros (pruebas efectuadas con it madres). del lactante entre el segundo y el octavo dia postnatal. En cada prueba olfativa, la madre debe escoger entre tres camisetas que ban sido llevadas por tres lactantes diferentes; en cada prueba. acústica, la madre debe escoger entre grabaciones de lloros de varios recien nacidos, entre los cuales se encuentra el suyo.

Durante este mismo periodo se ha observado en la madre la evolucion de la eliminación urinaria de los 17 -OHCS.

mamiferos llamados «macrosmáticos» (que tienen una sensibilidad olfativa muy desarrollada). Ahora bien, se sabe que existen relaciones anatomo-funcionales muy estrechas entre las células sensoriales de la mucosa olfativa y el sistema limbico, cuyo papel es muy importante dentro de los comportamientos telacionales de los mamiferos (P. Karli, 1976-1982).

Dichas bases olfativas subsisten luego del periodo neonatal hasta la edad de, años (H. Montagner, 1974). Ciertos autores sugieren asimismo que los vínculos afectivos que establecemos en la edad adulta dependen de nuestras experiencias olfativas precoces. Asi, J. Bowlby (1969-1973) piensa que a partir de dichos indicios (olfativos, táctiles, auditivos...) surgen los sistemas altamente discriminativos y sofisticados que más tarde, en la infancia y en el resto de la existencia, mediatizan el apego hacia figuras y personas particulares. 
Parece entonces primordial favorecer los intercambios madre-bebé a través de todos los canales sensoriales, incluido el canal olfatorio.

Resalta que los intercambios que alimentan el vinculo entre la madre y el niño no se limitan únicamente a los actos recíprocos del comportamiento visible, los intercambios de olores, el calor corporal, el sabor de la leche materna, el ritmo cardíaco, las entonaciones vocales, los contactos mutuos, constituyen los «lenguajes secretos» de las primeras relaciones que se establecen entre la madre y el niño pequeño. Podemos entonces preguntarnos si la ausencia o la perturbación de las relaciones olfativas entre el recién nacido y su madre no acarrearian disturbios en el desarrollo afectivo del niño (niño abandonado o separado de la madre temporalmente).

\section{Ontogenia de las secuencias de comportamiento en el niño de 6 a 36 meses}

Con posterioridad a los estudios sistemáticos efectuados por nuestro equipo de investigación en los niños de 2 a s años, realizamos la observación, desde 1975 , de otros grupos de niños desde la edad de 6 meses en adelante. Los métodos de investigación son los mismos descritos anteriormente, es decir: toma de apuntes, grabación de imágenes en filmadora super 8 o vídeo y grabaciọn sonora. El análișis de las secuencias de comportamiento y de la estructuración de las secuencias se hace por intermedio de peliculas que se analiza fotograma a fotograma, el análisis de las vocalizaciones se efectúa en un sonógrafo (gama de frecuencias, frecuencia fundamental, forma de la vocalización).

El análisis de la estructura de las secuencias de comportamiento en unidades más pequeñas llamadas «items», nos ha permitido observar que ciertos comportamientos, por ejemplo los items de vínculo y de apaciguamiento presentan una gran probabilidad de inducir en el niño receptor, respuestas de vinculo y de apaciguamiento, es decir, una sonrisa, una caricia, un contacto corporal, etc. Se han obtenido de esta manera unos coeficientes de correlación de 0,84 (en los niños de 8 a 26 meses) y de 0,96 (en los niños de 18 a 36 meses) entre los comportamientos de vinculo y de apaciguamiento y la totalidad de las actividades lúdicas.

Otros comportamientos, a menudo presentados a distancia, generalmente sin contacto corporal, inducen en más del 70 por too de casos el abandono de un objeto, el miedo, la fuga o el llanto del niño receptor. Estos items, llamados de amenaza, aparecen sobre todo en situaciones de conflicto y de competición por un objeto. Claramente diferenciados de las agresiones por su estructura y su función, dichos items pueden, sin embargo, agruparse dentro de la rúbrica de los comportamientos «agonísticos» como se hace generalmente en Etologia (Tabla I).

Los actos de apoderamiento se hallan fuertemente correlacionados con las agresiones (0,84 en los niños de ocho a veintisëis meses).

De esta manera se ha podido establecer una lista de las diferentes secuencias de comportamiento y de los items que las componen, al mismo tiempo que se ha estudiado el momento de su manifestación y 


\section{TABLA !}

Lista de los ítems de comportamiento que ban sido analizados y cuantificados en los niños de diecriocho a treinta y seis meses de edad (guarderia) y de tres a seis años (jardin de infancia).

Una secuencia se define como la combinatoria de dos o más ltems.

Los items y las secuencias de items son calculadas cada mes y cada año por cada niño.

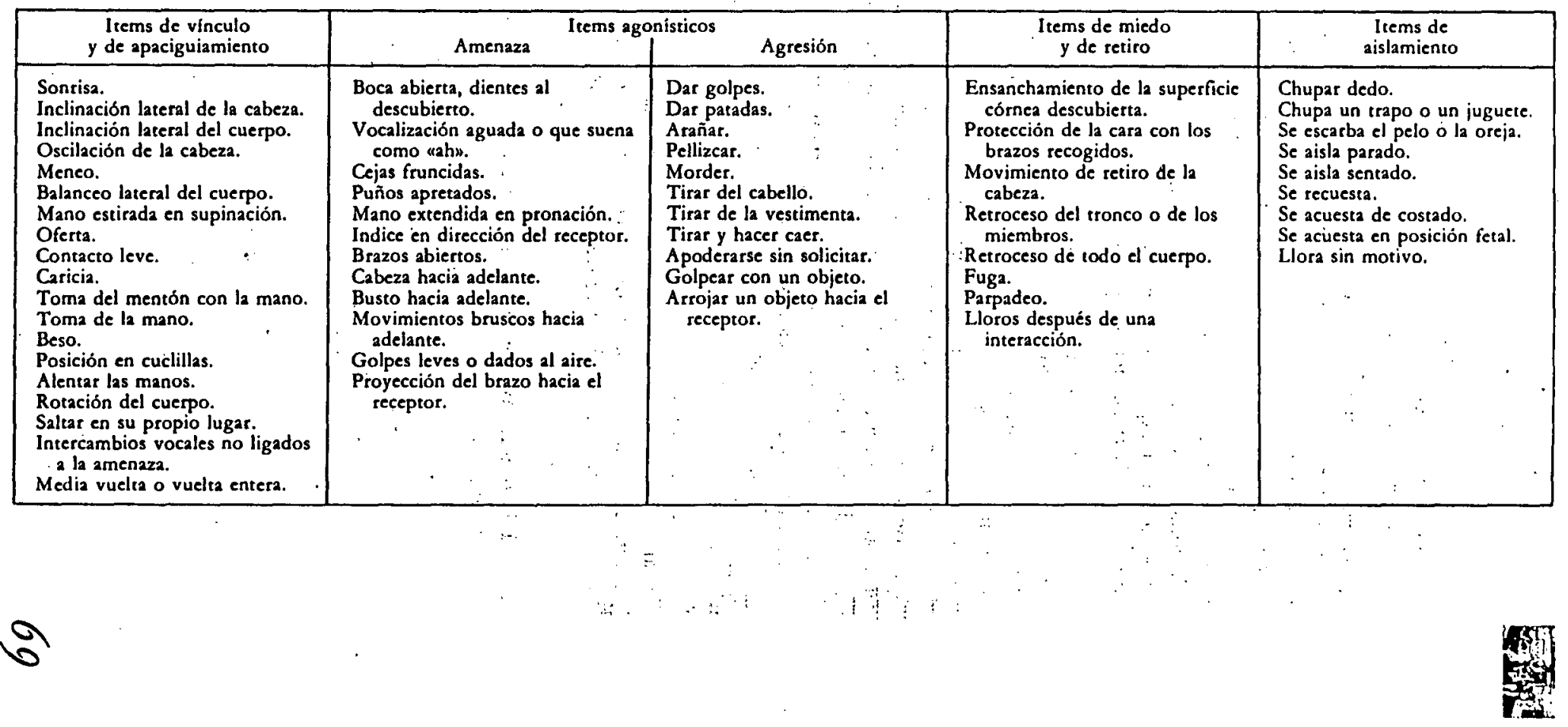


su desarrollo en cada niño. (Tabla ı). Asi, por ejemplo, desde un punto de vista ontogenético, los elementos de base de los comportamientos de amenaza (apertura de la boca acompañada de vocalización, avance del busto y de la cabeza, proyección de un brazo, balanceo lateral del cuerpo, mimica facial; etc.) aparecen y evolucionan de manera progre: siva con respecto a la edad del niño.

Es entre los nueve y los doce meses cuando aparecen ciertos items que forman parte de los comportamientos de amenaza para combinarse en, secuencias organizadas. Sin embargo, con respecto a otras categorias de comportamientos, la proporción de los comportamientos de amenaza representa 13 por 100 del total; entre doce y quince meses la proporción es de 2I,s por 100 . Dicha evolución temporal se encuentra relacionada con la adquisición de la marcha, la cual se acompaña de un aumento de la exploración del medio ambiente y paralelamente de un aumento de la frecuencia de los conflictos y de las competiciones.

Se considera que entre los veinticuatro y treinta y seis meses los diferentes items que componen las secuencias de la amenaza aparecen claramente organizados y diferenciados en la mayoria de los niños observados. La aparición precoz de los elementos de base de los comportamientos de amenaza permite de esta manera al niño pequeño participar en las competiciones con una escasa probabilidad de recibir agresiones. $Y$ se sabe que una de las principales funciones de la amenaza es precisamente la de prevenir la inminencia de una agresión dentro de una situación de competición o de conflicto. En la mayoría de los casos ( 59 por too) los comportamientos de amenaza van acompañados de una vocalización con una forma espectral característica. La frecuencia fundamental puede variar entre, 60 y $850 \mathrm{c} / \mathrm{s}$ o hertzios (Fig. s).

Otras secuencias de comportamiento (apaciguamiento; agresión, aislamiento, etc.) han sido igualmente estudiadas por nuestro grupo de investigación desde un punto de vista ontogenético y funcional (H. Montagner, A. Restoin, D. Rodríguez y col. 1978-1983).

\section{Diferenciación de los perfiles de comportamiento y ritmos biológicos"}

Las investigaciones realizadas a lo largo de trece años en nuestro laboratorio con niños de seis meses a cinco años, observados en guarderias y en jardines de infancia demuestran, cada vez más claramente, que las relaciones entre niños de la misma edad se hallan reguladas por intercambios de mímicas, posturas, gestos, contactos corporales y vocalizaciones poco variables de un niño a otro y de una población a otra. Todos los items y secuencias de comportamiento descritos se manifiestan en el niño al sexto y el quinceavo mes, salvo los comportamientos directamente ligados al dominio de la marcha, a la adquisición de la supinación y al desarrollo de la motricidad final del rostro. Los elementos motores que componen dichos tipos de intercambios parecen entonces constituir los «universales» de la especie humana, sin que, por tanto, se pueda establecer un origen genético. Sin embargo, la no aparición de dicho nivel de organización o la aparición de comportamientos diferentes durante las interacciones entre un niño 
(D)
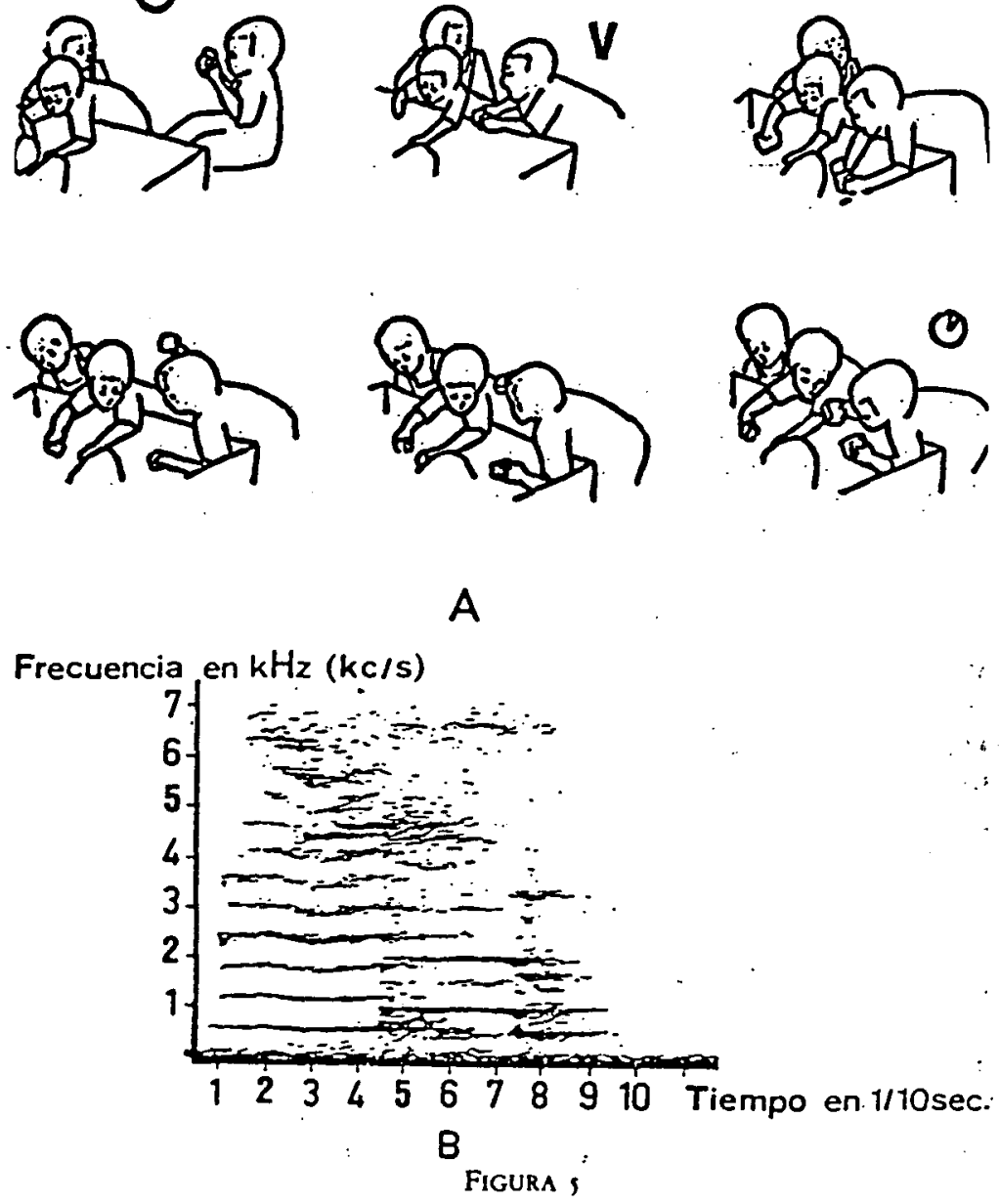

Ejemplo de vocalización caracteristica (B) emitida por el niño $V$ (12 meses) durante un comportamiento de amenaza caracterizado al mismo tiempo por la proyección de la cabeza y del busto en dirección del reseptor. El niño $(V)$ se encuentra en situación de competición para tener acceso a una caja donde se hallan varios juguetes. El espectrograma (sonograma) de la vocalización de amenaza se caracteriza por una frecuencia fundamental que varia entre 560 y $890 \mathrm{c} / \mathrm{s}$ (media: $700 \mathrm{c} / \mathrm{s} \pm 13 \mathrm{~s})$. Lo duracion es breve ( $\not{q} / 10$ sec.) y su forma es caracteristica (armónicos dispatestos borizontalmente).

con sus semejantes, o la no estructuración en secuencias significativas, podrian constituir unos indicios precoces de una probable evolución de aquel niño hacia un estado patológico.

Cuando se cuantifican de un mes a otro los porcentajes relativos de las categorias de comportamiento inventariadas $y$, cuando se analiza la estructura de sus encadenamientos durante las interacciones del niño con sus semejantes, se observa que ciertas tendencias comportamentales se manifiestan claramente en la mayoría de los niños en el transcurso del segundo año. De manera particular la frecuencia de aparición de las 
diversas secuencias de comportamiento se vuelve diferente de un niño a otro. El análisis factorial de correspondencias muestra que, en situación de emisor, ciertos niños se diferencian claramente de los demás (figura 6). Efectivamente la unube negra» dentro de la cual se inscriben los resultados de las observaciones hechas en cada uno de los 10 niños de ocho a veintiséis meses se estira hacia los ejes de vinculo y de atracción (ejes 3 y 4) para el niño E; hacia los ejes de las agresiones y de las interacciones no determinadas, sin significado o función claros (ejes 2 y i) para los niños F y J; y hacia los ejes de los temores (eje s) para el niño $G$ (la nuble clara, que abarca la nube negra representa la suma de los resultados obtenidos en los 10 niños.

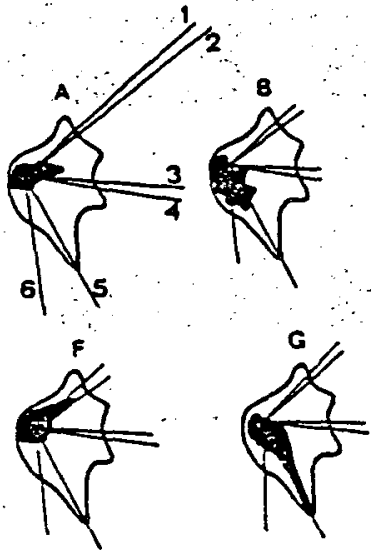

1. Interacciones no determinadas

2. Agresiones

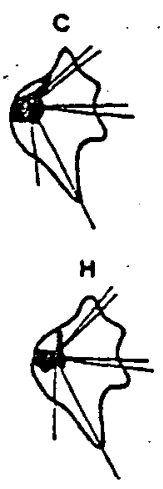

3. Vinculos

4. Atracciones
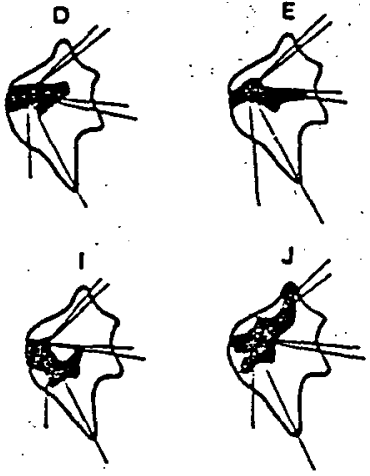

5. Temores

6. Repliegues

Figura 6

Representacion gráfica del análisis multidimensional de los diferentes caracteres de comportamiento que ban sido escogidos en cada niño ( $A$ a $J$ ) con respecto a los mismos caracteres presentadas por el conjunto de niños estudiados $(N=10)$. La nube negra delimita la cantidad de observaciones efectuadas en cada niño. La nube clara delimita la cantidad total de observaciones efectuadas en los 10 niños. Los gjes 1 a 6 definen los caracteres de comportamiento de la Tabla i a los cuales se ba adjuntado la categoria winterccciones no. determinadasw (sin significado o función precisos). La edad de los niños varia entre ocho:y veintiséis meses. Se denota, por ejemplo, en los gráficos la proximidad de los ejes de los vinculos y de las atracciones y aquellos de las interacciones no determinadas $J$ las agresiones.

Todo esto muestra que, en el transcurso del segundo año, un segundo nivel de organización de los comportamientos relacionales se estructura tras un proceso de «diferenciación»'y de «selección» realizado por el niño dentro de su repertorio motor.

Dicha tendencia, al reforzarse durante el tercer año, hace posible distinguir varios perfiles comportamentales, tomando en cuenta las proporciones relativas de las diferentes categorias de comportamiento, la resultante de los fracasos y éxitos en las competiciones y la estructura del encadenamiento de las secuencias comportamentáles (H. Montagner y col. 1978). 
comportamentales: perfil lider (niño actractivo y apaciguante), dominante agresivo (presenta más agresiones que actos de vinculo y de apaciguamiento), dominante fluctuante (alterna los comportamientos del lider y del dominante agresivo), dominado con mecanismos de lider (estructura comportamental del lider), dominado temeroso (los items de temor son frecuentes), dominado agresivo (alterna agresiones, temot y aislamiento), dominado aislado (niño solitario y reprimido). Pero la evolución hacia tal - cual perfil de comportamiento depende principalmente del modo relacional que se establece entre el niño y su medio sociofamiliar, sobre todo con su madre.

La recolección de ciertos datos fisiológicos, de informaciones sobre el medio familiar y sobre el ritmo de vida del niño (ritmo sueño-vigilia, ritmos escolares) nos ha permitido delimitar las variables que influencian las modificaciones del perfil comportamental entre el segundo y el quinto año de la vida del niño. Se ha analizado de manera particular las curvas circadianas (del latín "circa» cerca y "dies» un día) de eliminación urinaria de las hormonas secretadas por la corteza de las gländulas suprartenales (cortisol y 17 :OHCS).

El resultado de los análisis de dichos metabolitos urinarios confirma la existencia de correlaciones entre la estructura del comportamiento relacional (perfil comportamental), la estructura y la variabilidad de las curvas circadianas de los 17 -OHCS.

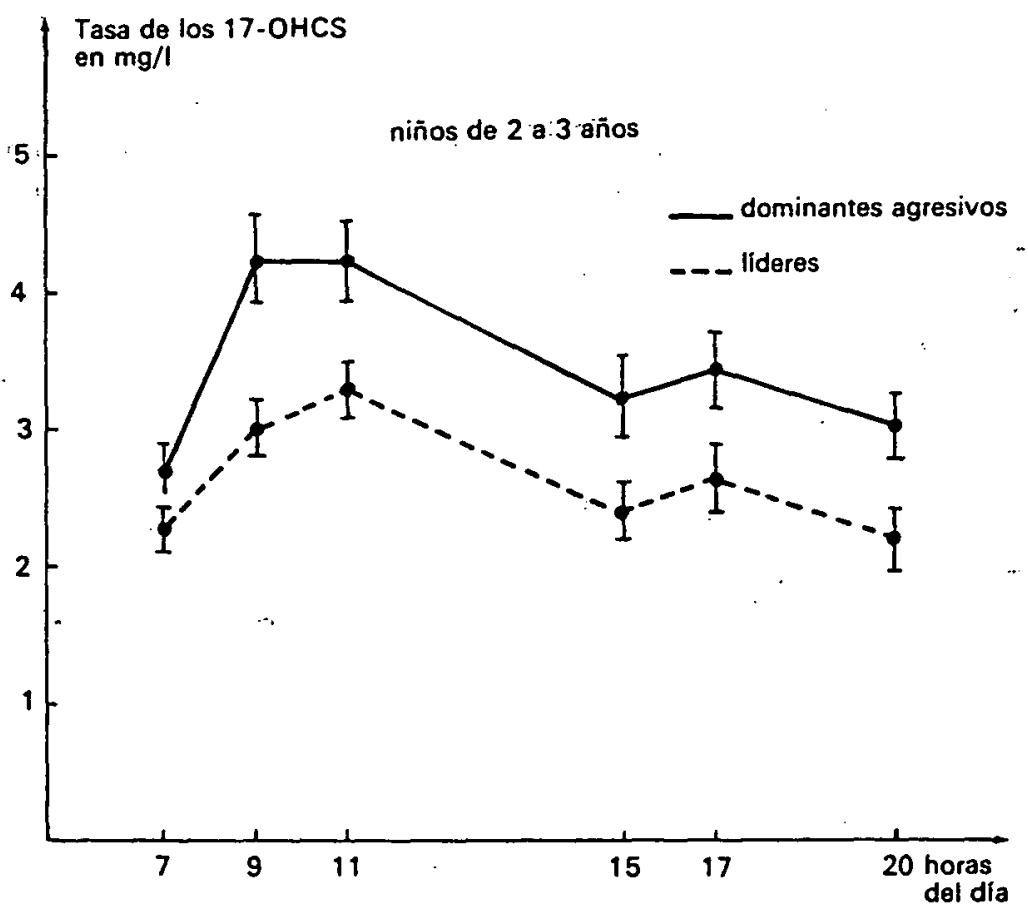

Figura 7

Comparación entre las curvas circadianas medias de 17 niños lideres $y$ de 16 niños dominantes agresivos de dos a tres arios de edad. Se nota una diferencia entre las tasas de secreción de los 17-OHCS arinarios seguin el perfil comportamental de los niños. Las diferencias entre las curvas medias son significativas a $p<0,01$. 

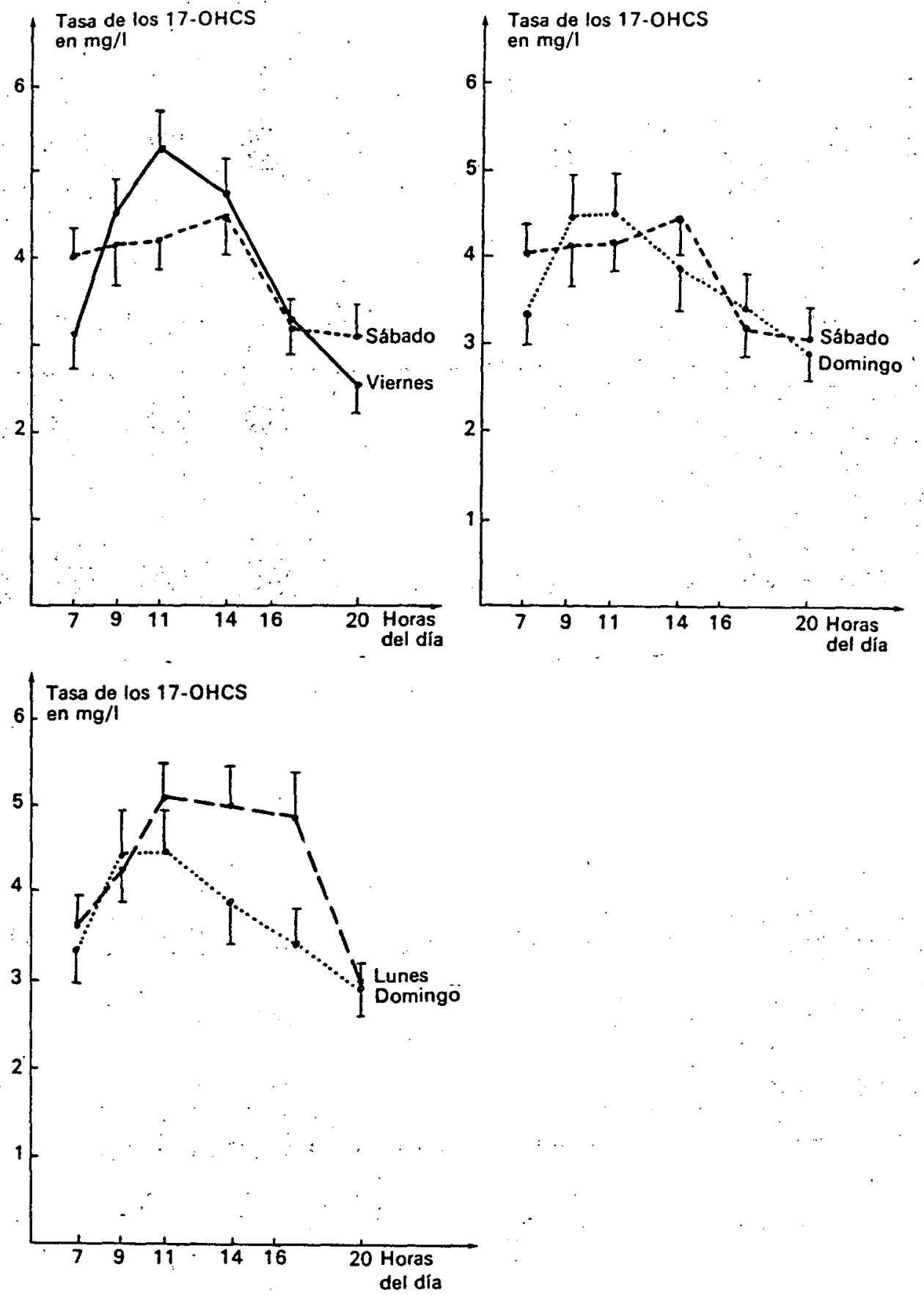

Figura 8

Comparación entre las curvas circadianas medias de los $17-$ OHCS de 26 niños de tres a cuatro años de edad, observados en el jardin de infancia. Se nota una diferentia entre las curvas correspondientes a los dias, viernes, sábado, domingo y lunes. La curva media del dia lunes se mantiene en un nivel elevado. La curva del dia sábado presenta a las 1 horas un aumento que corresponde a la llegada del niño al medio familiar (en Frantia las slases se terminan el sábado a mediodia). El cambio de ritmo de vida del niño se acomparia entonces con una desincronizasión de las curvas de los 17-OHCS. 
En la guarderia, la variación de la tasa de los 17 -OHCS de un dia a otro depende de la tendencia en el niño a privilegiar los comportamientos de agresión hacia sus semejantes. En cambio los niños que presentan una tasa estable de eliminación de los 17-OHCS son igualmente estables en sus comportamientos relacionales (lider, dominado con mecanismos de líder). Por ejemplo, un niño de tres años de tipo lider presenta generalmente una curva circadiana media de los $17-\mathrm{OHCS}$ tipica y regular: un pico hacia las I I a.m., seguido de una baja progresiva hasta la noche (figura 7 ). Un niño agresivo presenta por su parte una curva irregular con una tasa alta de eliminación de los 17-OHCS (figura 7).

Por último, los cambios de ritmo de vida en el niño se acompañan con una perturbación de su comportamiento y una alteración de la tasa de eliminación de dichos metabolitos. La ruptura de ritmo de vida que constituye el retorno del niño, el sábado, de la escuela al medio familiar, induce una desincronización de su comportamiento y de su fisiología. Inversamente, el retorno del niño, el lunes, de la familia al medio escolar, se mediatiza con las mismas perturbaciones. La figura 8 nos permite comparar las curvas circadianas medias de los 17 -OHCS de 26 niños franceses observados en un jardín de infancia. Se puede constatar que las curvas de los dias sábados y lunes (días de cambio de ritmo de vida para el niño y la familia) presentan un nivel más elevado de eliminación de los 1.7 -OHCS que los dias viernes y domingo.

Resalta de dichos estudios que los cambios de ritmo de vida en el niño, impuestos por la familia y el medio escolar, se convierten en àlteraciones de su comportamiento y de su fisiología. A estos factores puede añadirse la actirud del medio familiar y escolar hacia el niño y que puede ser susceptible de acentuar $o$, al contrario, de atenuar dichas perturbaciones.

\section{CONCLUSION}

Lejos de querer oponerse a los estudios de Psicologia experimental, de Psicología del niño, de Psicología clinica, de Psicologia social o de Antropología, las investigaciones en Etologia humana pueden aportar complementos apreciables para dichas disciplinas sobre todo por la renovación de la metodología y de sus técnicas de observación y de análisis de los comportamientos.

El establecimiento de los primeros vinculos afectivos entre la madre y el recién nacido depende de la manera en que se realiza el intercambio de las informaciones auditivas, visuales, olfativas y somestésicas vehiculadas por los canales sensoriales.

Hay que subrayar de manera particular el papel del olfato como factor de regulación de los intercambios madre-lactante. Dichos estudios nos permiten delimitar el papel respectivo de los diferentes tipos de estímulos sensoriales a partir de los cúales se construyen, se refuerzan o se deterioran los intercambios entre la madre y el niño durante tal o cual periodo de la vida posnatal.

Paralelamente, el estudio en continuo de los sistemas de comunicación del niño con sus semejantes, desde la edad de seis meses en 
adelante, muestra que al final del primer año de vida los niños disponen de un repertorio común de gestos, mimicas, posturas y vocalizaciones que parecen constituir los "universales» de la especie humana, sin que, por tanto, se pueda determinar un origen genético.

Durante el segundo año se manifiestan en el niño unas tendencias comportamentales mediante un proceso de diferenciación y de selección de ciertos tipos de comportamientó. Es asi como el niño dentro de sus relaciones con sus semejantes tenderá en privilegiar ciertos comportamientos de agresión o, al contrario, ciertos comportamientos de apaciguamiento. Dichas tendencias comportamentales, al reforzarse en el transcurso del segundo y del tercer año, caracterizan en el niño un perfil de comportamiento. No obstante, la diferenciación de dicho perfil se halla directamente ligado al modo de relación de la familia, preferencialmente de la madre, con el niño y esto desde temprana edad (A. Restoin, H. Montagner, D. Rodriguez y col. 1983-1984). Paralelamente, la dosificación de ciertas hormonas secretadas por la corteza de las glándulas suprarrenales ha demostrado la existencia de correlaciones entre la estructura del comportamiento relacional (perfil comportamental), la estructura y la variabilidad de las curvas circadianas de los I7-OHCS (metabolitos urinarios del cortisol).

Todos estos datos confirman que sólo los estudios integrados de la ontogenia de los sistemas de percepción, de los comportamientos y de los ritmos biológicos, permitirán precisar mejor el papel de los estimulos exógenos en el desarrollo sensorial, relacional y afectivo del niño. El estudio etológico del desarrollo humano es importante no solamente porque él puede satisfacer nuestra curiosidad cientifica, sino tambièn y sobre todo porque sus resultados pueden cambiar la manera de tratar al niño.

\section{Resumen}

Al abordar al estudio del comportamiento bumano, los etólogos ban contribuido a la renovación de los printipios y metodos de investigación sobre los sistemas de comunicación en el bombre.

El grupo de investigacion de la Universidad de Besanfon ba estudiado la aparicion, las modificaciones y las funciones de la comunicación no perbal en el niño desde el nacimiento basta la edad escolar. Durante el periodo posnatal, el estudio de las interacciones olfativas y acxisticas entre la madre y el niño ba demiosirado que el comportamiento del lactante se bala modificado desde el tercer dia por la percepción del olor materno. Al mismo tiempo el recomocimiento, por la madre, del olor y de la voz del recién nacido sigue wna evolucion similar.

Mas tarde, mediante el estudio de los sistemas de comuinicación del nisio con sus semejantes se ba demostrado que basia la edad de doce meses, el niño utiliza un repertorio de "universaless de comportamiento. En el transcurso de segundo año, los niños combinan aquellos asniversales" de manera diferente para asi establecer un estilo relacional propio o- "perfil comportamentaly. Por uiltimo, existe uns correlacion estrecha entre el aperfil comportamentals y las variaciones fisiológicas del niño.

\section{Summary}

In dealing sitb buman bebaviour, etbologists bave contributed with renewing principles and research methods on communication systems in man.

The University of Besanfon researsb group has carried out investigations on the emergence, changes and functions of non-verbal communication in cbildren since birtb until tbe scbool age. The investigation of olfactory and acustic interactions between motber and cbild during postnatal period sbows evidence of modifications of baby bebaviour, starsing in the third day of life and due to tbe motber smell. A similar evolution develops in tbe motber respecting the smell and voice of the new born.

It has also been prooved tbrough the study of the communication system that later on, when the baby is about twelve montbs old, the cbild uses a repertory of aniversalsw of bebaviour. During the second year children combine sbese amiversalsw in a different manner, establisbing tbis way a personal style or bebavioural profile in tbeir relations. Finally, a close correlation is beld between tbe wbebavioral profiless and sbe pbysiological variation in cbildsen. 
En abordant lítude du comportement bumain, les äbologistes ont coniribué as renouyellement des démarcbes et des methodes de recbercbe sur les systimes de communication chez 1 Homme.

Le groupe de recbersbe de PUniversité de Besanfon a eiudie Temergence, les modifications et les fonctions de la communication non verbale cbez fenfant depuis la naissance jusqu'al äge scoleire. Pendant la période post-natale. Pitude des interactions olfactives at acoustiques entre la mere et lenfans a montri que le comportement du nourrisson est modifie a partir du je jour par la perception de fodeur matcrmel. En meime semps la reconaissance par la mere, de lodeur es de la voix du nowvean-nè suit une evolution similejre.

Plus tard, par fítude des gytimes de communication de lenfant avec ses pairs an a montre que vers lige de 12 mois, renfant utilise un répertoire dinuniversauxp de comportement. An cours de la $2 \dot{e}$ anné, les anfants combinent ces "universaux" de fofon différente pour ilablir ainsi un style relationnel propre os oprofil de comportements. Enfin, il existe una corrilation étroite entre le aprofil de comportement" et les variations pbisiologiques cbez renfant.

\section{Referencias}

A juriaguerra, J. de: "Ontogenèse de la motricitè. En A. Hecaen y M. JeAnNerod: Du contrôle moteur à Porganisation du geste. París, Masson. 1977.

Blurton Jones, N. G.: Ethological studies of child behaviour. Cambridge, Cambridge University Press. 1972.

BOwER, T. G. R.: Development in infang. San Francisco, Freeman. 1974.

BOWER, T. G. R.: Le developpement psyebologique de le premiëre enfonce. Bruselas, Mardaga. $197^{8 .}$

Bowlby, J.: Attacbment and loss. Vol. I, Attachment. Nuev2 York, Basic Books, 1969. Ibid. Vol. II, Separation, Anxiety and Anger. Londres, Hogarth. 1973.

Buliow A, M. (Ed.): Before speecb. Cambridge, Cambridge Univ. Press.

EIBEL-EIBESFELDT, I.: "Human ethology: concepts and implications for the sciences of manm. The bebavioral and brain sciences, 1979, 2: 1-57.

Garrigues, P.; Mennesson, J. F.: Gulllon, J.; Visier, J. P., y Montagner, H.: wFluctuations comparées de l'activité motrice chez des enfants normaux et des enfants retardés observés en siruation de jeum. En H. Montagner: Les gitbmes de Penfant ef de ladolescent. Paris, Stock. 1993.

HINDE, R. A.: Biological basis of buman social bebayiour. Nueva York, Mac Graw Hill. 1974 .

LEwIS, D.: Tbe secret language of your cbild. Londres, Souvenir Press. $197^{8}$.

LIPSITT, L. P.: *The study of sensory and learning process of the new born». Clinies in Perinatology, 1977, 4: $163-186$.

MAC FARLANE, A.: "Olfaction in the development of social preferences in the human neonates". Cibo Foundation Symp, 33. Amsterdam, Elsevier. 197s.

MAC GREW, W. C.: Etbological study of cbildren's bebavior. Nueva York, Academic Press. 1972.

MELhER, J :: «La perception du langage chez le nourrisson*. La Recbercbe, 1978, 88.

Millot, J. L.; Filiatre, J. C., y MONTAgner, H.: "Etudes préliminaires des comportements de toucher portés par la mère à son nouveau-nen. En Le bébé dans un monde ett cbangement, Second Congrés Mondial de Psychiatrie du Nourrisson. Paris, Ed. INSERM. $19^{8} 3$.

MONTAGNeR, H.: "Communication non verbale et discrimination olfactive chez les jeunes enfants Approche èthologiquen. En E. MORIN y M. PIATtelu: L'unité de l Homme. Paris, Le Seuil. 1974.

Montagner, H.; Arnaud, M.; Jeandroz, J.; Renner, N.; Henry, E., y Henry, J. CH.: «Approche etho-physiologique des communications non verbales chez le jeune enfants. Colloque Intermational "Prilangagex. Besançon. 1973.

MONTAGNER, H., y HENRY, J. CH.: «Vers une Biologie du comportement de l'enfants. Revue des Questions Scientifiques, 1975, 146: $48 \mathrm{r}-529$.

Montagner, H.; Nenedini, M.; Bolzoni, D.; Henry, J. CH.; Lombardot; Moyse, A., y Rosier, M.: Mécanismes de la communication non verbale cbez les jesnes enfants. Film $16 \mathrm{~mm}$., sonido óptico, versión francesa e inglesa. Paris, Service du Film de Recherche Scientifique. 1976.

Montagner, H.; HenRy, J. CH.; LOMBardot, M.; Benedin!, M., y Restoin, A.: «Sur la différenciation des profils comportementaux chez les enfants de $i$ ì, ans ì partir de l'étude des communications non verbales». Psycbomotricite, 1977, $t:$; 3-88.

MONTAGner, H.; HenRY, J. CH., et cols.: «Etudes éthophysiologiques de groupes d'enfants de 14 mois à I ans à la crèche et à l'école maternellen. Etbologie bumaine. Psychologie Médicale, 1977, 9: $11,2075-2112$.

Montagner, H.; Henry, J. CH.; Lombardot, M.; Restoin, A.; Benedinl, M., y Moyse, A.: «Bchavioural profiles and corticosteroid excretion rythms in young children. Part I: Non verbal communication and setting up of behavioural profiles in children from 1 to 6 yearsn. En V. REYNOL.DS, y N. G., BluRton Jones: Humen Bebaviour and Adapiation. Londres, Francis \& Taylor, 207-228. Ibid., Part II: "Circadian and weekly rythms in corticosteroid excretion levels of children as indicators of adaptation to social contexen. En V. REYNOLDS y N. G. BluRTON JONEs: Human Bebaviowr and Adeptation. Londres, Francis \& Taylor, $229-265.1978$.

MONTAGNer, H., y HENRY, J. CH.: «Apport des méthodes étho-physiologiques à l'étude des conduites humainesn. Coloquio "Le cervean âge et ses medicaments. $197^{8}$.

Montagner, H.: L'enfont et la communication. Paris, Stock, 1978. (Traduc. cast. en preparación. Madrid: Visor / Aprendizaje.)

MONTAGNER, H.: "L'éthologie de l'enfant». Le comportement animal. N.o Spécial «Science et Vie», 1978, 125: $141-149$. 
Montugner, H.; Henry, J: CH.; Lombardot, M.; Restoln, A.; Godard, D., y BenediN, M.: "La différenciation des systémes relationnels, y compris les conduites d'agression au cours de l'enfance». VI th International Seminar in Comparative Clinical Criminology. Problemes Actuels en Criminologie Clinique. Recbercbes, diagnostique et Traitements. Montréal, 1978, 19-48.

Montagner, H.; Henry, J. CH.: Restoin, A.; LOMbardot, M.; Godard, D.; Bentini, M.; Rodríguez, D., y UILLMAN, V.: uLes comportements de communication et la notion d'adaptation chez le jeune enfantw. Actes du Congres National des Eroles Maternelles et Classes Enfantines Publiques. Poitiers, 6-19. 1978.

Montaǵner, h.; Henry, J. CH.; lombardot; M.; Restoin, A.; Benedini, M.; Godard, D., y Boili.ot, F.: whe ontogeny of communication behaviour and adrenal physiology in the young child». Cbild Abuse and Neglect; 1979, 3, 19-30.

Reingerg, A.; Fraisse, P.; leroy, C.; Montagner, H.; Pequignot, H.; Poulizac, H., y Vermeil., G.: L'Homme malade dx temps. Paris, Stock. 1979.

MONTAGnER, H.: «Ethologie Humaine». Enciclopaedia Universalis, 569-578. 1980.

Montagner, H.; Restoin, A.; Schaal, B.; Rodriguez, D.; Ullimann, V.; Ladouce, I.; Guedira, A., y VIAI.A, M.: "Apport éthologique à l'étude ontogénétique des systèmes de communication de l'enfanos. Ecologie Humaine, 1981, 39. 1445 B, 3906-3922.

Montagner, H.: «Données nouvelles sur les systèmes d'interaction entre le noutrisson. et sa mères: Neuropsycbiatrie de $/$ Enfance, $1982,30,153-158$.

Montagner, H.; Restoin, A, y Henry, J. CH.: "Biological Defense Rythms, Stress and Communicationn. En W. W. HARTUP: Revien of Child Development Research. 1982. 6, 291-319.

Montaginer, H.; Restoin, A.; Godard, D.; Viala, M.; Rodriguez, D.; Ullmann; V., y Millot, J. L.: «Les communications non verbales et la structure des groupes chez les jeunes enfantsw: En G. Azemar y H. Ripolli: Elements de Neurobiologie des Comportements Moteurs. Paris, Ed. de L'INSEP, 247-269. 1982.

Montagner, H.: Les Rytbmes de /'Enfant et de P'Adoleseent. Paris, Stock. 1983.

Montagner, H.; Restoin, A.; UllmanN, V.; Rodriguez, D.; Godard, D., y Viala, M.: “Development of early peer interaction». En W. DOISE y A. PAl.monarl: Social Interaction in Individial Development. Cambridge, Cambridge University Press. 1983. (En prensa.)

Montagner, H:; Restoin, A.; Laurent, D.; Rodriguez, D.; Ullmann, V., y' Viala, M.: ales comportements agonistiques et leur genèse chez l'enfant». Pour un sport sans violence. París, Pergamon Press, 33-43, 1983 .

MONTAGNER, H.: "Observation du comportement et èthologie humaine - Le nourrisson et le jeune enfant». Le bébé dans un monde en ebangement, Second Congrés Mondial de Psychiatrie du Nourrisson. Paris, Ed. INSERM. 1983. (En prensa.)

Montagner, H.; Restoin, A.; Laurent, D.; Rodriguez, D.; Ullmann, V., y Viala, M.: "Contribution à l'étude de la genèse des systèmes de communication du jeune enfant avec ses pairs". Le bébé dans un monde en changement, Second Congrés Mondial de Psychiatrie du Nourrisson. Paris, Ed. INSERM. 1983. (En prensa.)

Montagner, H.; Restoin, A.; Rodriguez, D.; Ullmann, V.; Viala, M.; laurent, D., y Godard, D.: "Social interactions of young children with peers modifications in relation to environmental factorss. En M. R. A. Chance: Social Cobesion in Monkeys, Apes and Humans. Nueva York y Londres, John Wiley and Sons. (En prensa.)

ReinberG, A., y Ghata, J.: Les Rytbmes Biologiques. Paris, uque sais-je?» Press Universitaires de France. 1978.

Restoin, A.; Rodriguez, D.; UllmanN, V., y MONTAGNer, H.: wLes systèmes de communication du jeune enfant avec ses pairs". Les premières années de la. vie. Especial «Science et Vie», 1983.

Restoin, A.; Montagner, H.; Gonard, D.; Henry, J. CH.; Henrotte, J. G.; Lombardot, M., y BENEDINI, M.: $\alpha$ New data on eircadian rythms of corticosteroid hormones and magnesium in the young child in the day care centre and in the kindergarten". En A. REINBERG, N. VIEUX y P. ANDLAUER: Night-and Sbift Work-Biological and Social Aspects. Oxford, Nueva York, Paris, Pergamon Press. 1981.

Restoin, A.; Rodriguez, D.; Ullmann, V., y MONTAGner, H.: uDonnćes nouvelles sur l'étude ontogénétique des séquences comportementales chez l'enfant". Erologie et Ethologie bumaines. 198 2. $1,41-6,9$.

Restoin, A.; Montagner, H.; SChanl, B.; Viala; M.; Ladouce, I.; Rodriguez, D.; Guedira, A.; Ullmann, V., y Millot, J. L.: «Le développement sensoriel, relationnel et affectif de l'enfanos. Education Pbysigue et Sportive en Ier degré, 1981, f; 1982, 6; 1982,7.

Restoin, A.; ROdrigueż, D.; UllimanN, V., y MONTAGNER, H.: *Données nouvelles sur l'ontogenèse

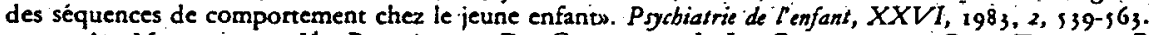

Restoin, A.; Montagner, H.; Rodriguez, D.; Girardot, J. L.; Casagrande, C., y Talpain, B.: «Données nouvelles sur le développement chronologique des systèmes de communication du jeune enfant avèc ses pairss. En R. PAGes: Recbercbes en Psychologie Sociale. Paris. (En prensa.)

Restoin, A.; MONTAgner, H. Rodriguez; D.; LAurent, D.; KONTAR, F., y. Ullalann, V.: " Données nouvelles sur la chronologie des comportements de communication et sur les profils de comportement chez le jeune enfanon. En R. TREMBLEY y col.: Developpement de l'enfant et Ethologie. Montreal. (En prensa.)

RODRiguez, D.: Contribution à l'étude ontogénetique des séquences de comportement et des vocalisations chez le jeune enfant. (Tesis doctoral aNeurosciences». (En preparación.)

Rodriguez, D.; Montagner, H.; Restoln, A.; SchaAl, B., y Ulimann, V.: aAporte de la Etologia al estudio de los Sistemas de Comunicación No Verbal en el Niñom. Actas del XIX Congreso Interamericano de Psicologia. México, Ed. Trillas. (En prensa.)

Rodriguez, D.; MONTAgner, H.; Restoin, A.; SchaAl, B., y Ullamann, V.: aAporte de la Etología al estudio ontogenético de los sistemas de comunicación no verbales en el niñon. Psicologia del niño y del recién nacido. Cuadernos, n. 6, Univ. del Valle, Cali-Colombia. (En prensa.)

Rouchouse, J. C.: "Ethologie, ćthogramme et communication non verbale nourrissons". Enfance, $1978, r$.

RufFIE, J.: De la Biologie à lo Culture. Paris, Champs-Flammarion. 1983. 
Schani, B.; Herlting, B.; MONTAGNer, H., y Quichon, R.: *Existe-t-il une communication olfactive entre la mère et son enfant nouveau-né-En E. Herbinet y M. C. Busnel.: L'aube des sens. Paris, Stock, 399.377 .1981 .

TRevarthen, C.; Hubley, P., y SCheeran, L.: «Les activités innées du nourrisson». La Recherche, 1971, 6.

ULLMANN, V.: «Contribution à l'etude ontogénétique du comportement et des rythmes biologiques chez le jeune enfant à la crechew. Tesis Dastoral «Biologie du Comportementu, Université de Besançon. 